University of Wollongong

Research Online

Faculty of Engineering and Information

Faculty of Engineering and Information

Sciences - Papers: Part A

Sciences

2012

\title{
Performance analysis of STFC MB-OFDM UWB in WBAN channels
}

Miftadi Sudjai

University of Wollongong, ms917@uowmail.edu.au

Le Chung Tran

University of Wollongong, Ictran@uow.edu.au

Farzad Safaei

University of Wollongong, farzad@uow.edu.au

Follow this and additional works at: https://ro.uow.edu.au/eispapers

Part of the Engineering Commons, and the Science and Technology Studies Commons

Research Online is the open access institutional repository for the University of Wollongong. For further information contact the UOW Library: research-pubs@uow.edu.au 


\title{
Performance analysis of STFC MB-OFDM UWB in WBAN channels
}

\begin{abstract}
This paper investigates the performance of the space-time-frequency coded (STFC) multiband orthogonal frequency division multiplexing ultra-wideband (MBOFDM UWB) system over wireless body area network (WBAN) channels, using the Alamouti code. Different MIMO configurations and different modulations are used for comparison. The effect of body directions is also assessed by simulation in this paper. Simulation results show that the proposed STFC MB-OFDM UWB system provides a significantly better BER performance, compared to the conventional MB-OFDM system, even from a reasonably low Eb/No range. The simulations also confirm that the body direction affects the system performance significantly due to the availability of line-of-sight (LOS) or partial LOS paths. Overall, the STFC MB-OFDM UWB system can provide an energy efficient and robust, high data rate transmission for WBAN applications.
\end{abstract}

\section{Keywords}

$\mathrm{mb}, \mathrm{stfc}$, analysis, channels, wban, uwb, ofdm, performance

\section{Disciplines}

Engineering | Science and Technology Studies

\section{Publication Details}

M. Sudjai, L. Chung. Tran \& F. Safaei, "Performance analysis of STFC MB-OFDM UWB in WBAN channels," in 23rd IEEE International Symposium Personal, Indoor and Mobile Radio Communications (PIMRC 2012), 2012, pp. 1710-1715. 


\title{
Performance Analysis of STFC MB-OFDM UWB in WBAN Channels
}

\author{
Miftadi Sudjai, Le Chung Tran, and Farzad Safaei \\ School of Electrical, Computer, and Telecommunication Engineering \\ University of Wollongong, Australia. \\ Email:ms917@uow.edu.au
}

\begin{abstract}
This paper investigates the performance of the space-time-frequency coded (STFC) multiband orthogonal frequency division multiplexing ultra-wideband (MBOFDM UWB) system over wireless body area network (WBAN) channels, using the Alamouti code. Different MIMO configurations and different modulations are used for comparison. The effect of body directions is also assessed by simulation in this paper. Simulation results show that the proposed STFC MB-OFDM UWB system provides a significantly better BER performance, compared to the conventional MB-OFDM system, even from a reasonably low $E_{b} / N_{o}$ range. The simulations also confirm that the body direction affects the system performance significantly due to the availability of line-ofsight (LOS) or partial LOS paths. Overall, the STFC MBOFDM UWB system can provide an energy efficient and robust, high data rate transmission for WBAN applications.
\end{abstract}

\section{INTRODUCTION}

There have been active engagements in the body area network (BAN) research in recent years. BAN is a network of sensors or communicating devices placed in, on, or off the body to monitor physiological activities and motions. It is mainly driven by the need for more efficient and real-time health monitoring systems due to the aging society and the increasing cost of health care [16]. The development of advanced, tiny-sized, lightweight, and extremely low power implantable and wearable sensors are paving the way for the emergence of WBAN. In order to harmonize the developments of WBAN, IEEE set up a technical group TG6 within 802.15 to standardize the WBAN in November 2007.

Radio propagation in, on, and surrounding the human body is greatly affected by environment, posture, activities, and human tissue [12-20]. Numerous measurement campaigns on body centric propagations have been conducted to characterize the body centric channel, including ultra wideband (UWB) channel in the frequency bands of 3.1-10.6 GHz. Takada et. al. [12] show that the body centric channel varies due to the type of antennas, the position and orientation of antennas with respect to the body, the posture and motion of the body, and the variation of the human body itself. $\mathrm{Yu}$ Wang et.al. [14] claim that the body shadowing is a prominent factor in short-range body-to-body communications. The effect of locations of on-body devices, body size as well as the movement of the body is investigated in [17-20]. Finally, the IEEE 802.15 TG.6 has summarized and proposed a concept of WBAN channels [15] with four channel models CM1-CM4, which comprise seven scenarios (S1-S7). CM1 considers implant-to-implant link (S1) operating for medical information and communication science (MICS). CM2 determines implant-to-body surface (S2) and implant-to-external (S3) links operating in the same frequency band as CM1. CM3 considers body-to-body link, while CM4 considers body-toexternal link. Both are proposed to operate in several frequency bands, including UWB bands. Due to our focus on the UWB WBAN channel model, we will only consider CM3 and CM4 in this paper.

It is important to highlight the main differences between UWB channel models for Wireless Personal Area Networks (WPANs) [11] with the ones for WBANs [15], which are referred to as the UWB WBAN channel models hereafter. The UWB channel model in [11] is based on the Saleh-Valenzuela model without considering the effect of human body, while the UWB WBAN channel model [15] does. It is clearly shown in [15] that due to the shadowing effect of human body, the UWB WBAN channel produces a larger amplitude standard deviation $\sigma$ and a much greater exponential decay factor $\Gamma$, compared to the UWB WPAN channel. Moreover, $\Gamma$ varies significantly for different body directions with respect to the transmitter in the case of CM4 over the UWB WBAN channel. Therefore, it is necessary to investigate the performance of the MIMO MB-OFDM UWB system over these channels.

One of the emerging technologies for a short range, very high data rate communication is Multi Band Orthogonal Frequency Division Multiplexing (MB-OFDM) UltraWideband (UWB), endorsed by the WiMedia Alliance [22]. It combines the capability of OFDM to flatten the response of the dispersive-frequency selective channel of UWB, while maintaining the benefit of high capacity of UWB. It is designed to operate at up to $1 \mathrm{Gbps}$, with low cost and low power consumption.

Meanwhile, Multiple-Input Multiple-Output (MIMO) technology is claimed to increase the wireless system capacity significantly for the same total transmission power [1-8]. Its fundamental mechanism lies on the use of Space-Time Coding (STC) [1] which enables the data to be transmitted by multiple transmit (TX) antennas and received by multiple receive (RX) antennas. As a result, it enhances the diversity order and improves the link quality and capacity. In STC, signals are coded both in spatial and temporal domains. It is intuitive that further addition of the frequency domain processing in STC so that STC becomes Space-Time-Frequency Coding (STFC) provides better link performance, greater range, and higher data rate and system capacity. In [8], we have proposed the combination of MIMO, STFC, and MB-OFDM UWB, referred to as the STFC MB-OFDM UWB system, for general 
WPAN channels. This paper focuses on performance analysis of the implementation of STFC MB-OFDM UWB over WBAN channels using the Alamouti code.

There has been relatively intensive research on the MIMO OFDM system such as [2, 3], and on MIMO MB-OFDM UWB system including our previous works, for instances [410]. However, these systems were only implemented either in the frequency selective channel [2], Rician or Rayleigh fading channel [4], Nakagami-m fading channel [4], or UWB WPAN channel [5-10]. In [17, 18], Abbasi et. al. conducted performance simulation of MB-OFDM UWB system on their own channel models, rather than the IEEE channel model [15]. To the best of our knowledge, the performance analysis of a STFC MB-OFDM UWB system implemented in the proposed WBAN channels in [15] is still missing. Thus, this paper proposes the implementation of STFC MB-OFDM UWB in the WBAN channels using the Alamouti code. This code originally designed for frequency flat fading is capable of providing a full rate and a full diversity for up to two TX and two RX antennas [1]. However, it is obviously not suitable for the direct application to a very dispersive UWB WBAN channel. Therefore, in order to attain higher data rates and capitalize on the rich dispersion of UWB WBAN channels, a STFC MB-OFDM system can be deployed. Convolutional encoding and interleaver are included in the system to further reduce the effects of fading [21]. The bit error rate (BER) performance of the system is assessed through Matlab ${ }^{T M}$ simulations for two different MIMO configurations, i.e. 2I1O (two TX antennas and one RX antenna) and 2I2O (two TX antennas and two RX antenna). In addition, the performance is also compared for various M-PSK modulation schemes. Generalization for other MIMO configurations and other modulation schemes is straightforward.

The paper is organized as follows. Section II reviews the IEEE UWB WBAN channel models, which are different from the UWB WPAN channel model, as the basis to implement the system. Section III analyzes the proposed STFC MB-OFDM UWB. Simulation results and analyses are presented in Section IV. Section V concludes the paper.

\section{THE IEEE WBAN UWB CHANNELS}

Yazdandoost and Sayrafian [15] present the final document of the IEEE802.15.6 channel modeling subcommittee, providing channel models to be used in body area networks. The channel model aims to evaluate the performance of physical layer from various proposals and measurement campaigns.

The channel models are drawn basically from three possible types of nodes, namely implant nodes, body surface nodes and external nodes. Implant nodes are implanted below the skin or inside the human body. Body surface nodes are placed on the surface of the human skin, or at most $2 \mathrm{~cm}$ away, and external nodes are placed between a few centimeters and up to five meters away from the body. Fig. 1 shows the possible communication links between nodes and the channel model definition, i.e. CM1 - CM4. CM1 defines an implant-to-implant link (S1) operating in the MICS band

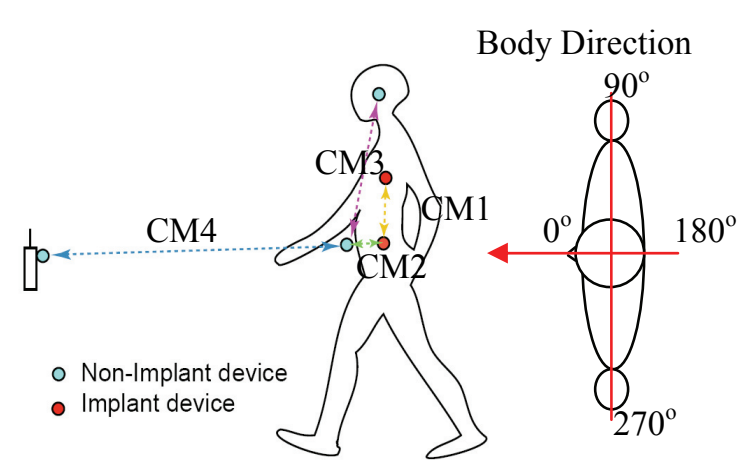

Fig.1. Possible communication links and channel models.

only (402-405 MHz). CM2 determines implant-to-body surface (S2) and implant-to-external (S3) links, operating in the same band as CM1. CM3 defines a body surface-to-body surface link for both LOS (S4) and NLOS (S5) scenarios. CM3 is intended to operate in seven different bands, including the UWB band $(3.1-10.6 \mathrm{GHz})$. CM4 determines a body surface-to-external link for both LOS (S6) and NLOS (S7). It is applied to three different frequency bands, including the UWB band. In this work, we only consider CM3 and CM4 for the UWB band.

The CM3 for the UWB band is derived from a measurement campaign in hospital room environment [15]. The channel response is characterized by a power delay profile (PDP) as follow

$$
h(t)=\sum_{l=0}^{L-1} a_{l} \exp \left(j \phi_{l}\right) \delta\left(t-t_{l}\right)
$$

where

and

$$
10 \log _{10}\left|a_{l}\right|^{2}= \begin{cases}0, & l=0 \\ \gamma_{0}+10 \log _{10}\left(\exp \left(-\frac{t_{l}}{\Gamma}\right)\right)+S & l \neq 0\end{cases}
$$

$$
\begin{gathered}
p\left(t_{l} \mid t_{l-1}\right)=\lambda \exp \left(-\lambda\left(t_{l}-t_{l-1}\right)\right) \\
p(L)=\frac{\bar{L}^{\mathrm{L}} \exp (\bar{L})}{L !}
\end{gathered}
$$

where $a_{l}$ is the path amplitude, $t_{l}$ is the path arrival time, and $\phi_{l}$ is the phase for the $l$-th path, respectively. $\phi_{l}$ is assumed to have an uniform distribution over $[0,2 \pi) . L$ is the number of the arrival paths and $\bar{L}$ is the average number of arrival paths. $\delta(t)$ is the Dirac function, $\Gamma$ is an exponential decay with a Rician factor $\gamma_{0}, S$ is a normally distributed random variable with zero-mean and standard deviation of $\sigma_{S}$, and $\lambda$ is the path arrival rate. The parameters of CM3 are presented in Table I.

TABLE I. PARAMETERS OF CM3.

\begin{tabular}{|l|l|l|}
\hline \multirow{3}{*}{$a_{l}$} & $\gamma_{0}$ & $-4.60 \mathrm{~dB}$ \\
\cline { 2 - 3 } & $\Gamma$ & 59.7 \\
\cline { 2 - 3 } & $\sigma_{S}$ & $5.02 \mathrm{~dB}$ \\
\hline$t_{l}$ & $1 / \lambda$ & $1.85 \mathrm{~ns}$ \\
\hline$L$ & $\bar{L}$ & 38.1 \\
\hline
\end{tabular}


The CM4 model is based on office environment measurements in which the TX antenna is fixed near to the wall, while the Rx antenna is placed on body and varied for different positions [15]. The effect of ground is considered in measurements. The channel response is characterized by a PDP as below

$$
h(t)=\sum_{m=0}^{L-1} \alpha_{m} \delta\left(t-\tau_{m}\right)
$$

where

$$
\left|\alpha_{m}\right|^{2}=\Omega_{\mathrm{o}} \mathrm{e}^{-\frac{\tau_{\mathrm{m}}}{\Gamma}-k[1-\delta(m)]} \beta
$$

$k=\Delta k\left(\frac{\ln 10}{10}\right) ; \tau_{o}=d / c ;$ and $\beta \sim \log \operatorname{normal}(0, \sigma)$

where $L$ is the number of arrival paths, modeled as a Poisson random variable with the mean value of $400, \alpha_{m}$ is the amplitude of each path. $\tau_{m}, m=1, \ldots, L-1$, is timing of path arrivals and is modeled as a Poisson random process with the arrival rate $\lambda=1 / 0.501251 \mathrm{~ns}, k$ is the K-factor (NLOS), $\Omega_{0}$ is the path loss, $d$ is the TX-RX distance, and $c$ is the velocity of light.

The parameters of CM4 also depend on the direction of body toward the TX antenna, and listed in the Table II.

TABLE II. PARAMETERS OF CM4.

\begin{tabular}{|c|l|l|l|}
\hline Body Direction & $\Gamma(n s)$ & $k(\Delta k(d B))$ & $\sigma(d B)$ \\
\hline $0^{\circ}$ & 44.6364 & $5.111(22.2)$ & 7.30 \\
\hline $90^{\circ}$ & 54.2868 & $4.348(18.8)$ & 7.08 \\
\hline $180^{\circ}$ & 53.4186 & $3.638(15.8)$ & 7.03 \\
\hline $270^{\circ}$ & 83.9635 & $3.983(17.3)$ & 7.19 \\
\hline
\end{tabular}

\section{STFC MB-OFDM UWB SYSTEM}

Fig. 2 depicts the block diagram of our proposed STFC MIMO-OFDM UWB system [8] with $M$-TX antennas and $N$ RX antennas. The data stream $d(n)$ is convolutionaly coded and interleaved, before being mapped to symbols. The modulation symbol stream is then converted by a Serial-toParallel (S/P) block into the symbol blocks (or vectors) $\overline{\mathrm{x}}_{t, m}=\left[x_{t, m, 1}, x_{t, m, 2}, \ldots, x_{t, m, N_{f f t}}\right]^{T} . N_{f f t}$ is the size of FFT.

The STC block creates space-time codes for the two consecutive symbol blocks by using the Alamouti code [1] as follows

$$
\mathbf{X}=\left\{\overline{\mathrm{x}}_{t, m}\right\}_{T \times M}=\left[\begin{array}{cc}
\overline{\mathrm{x}}_{t, 1} & \overline{\mathrm{x}}_{t, 2} \\
-\overline{\mathrm{x}}_{t, 2}^{*} & \overline{\mathrm{x}}_{t, 1}^{*}
\end{array}\right]
$$

where $\bar{x}_{t, 1}$ and $\bar{x}_{t, 2}$ are the symbol vectors transmitted from the first antenna and the second antenna at a given time slot, respectively. ( $)^{*}$ denotes complex conjugate. The symbol vectors in the columns of matrix $\mathbf{X}$ are transmitted simultaneously from $M$ different TX antennas, while elements in the rows of matrix $\mathbf{Y}$ are transmitted in $T$ different OFDM time slots $(M=2$ and $T=2$ for the Alamouti code) from the respective antenna. Each of symbol vectors in the matrix $\mathbf{X}$ is then converted into an $N_{f f t}$-point MB-OFDM symbol by the IFFT block, resulting in the STFC code matrix $\mathbf{X}_{\text {OFDM }}$ whose

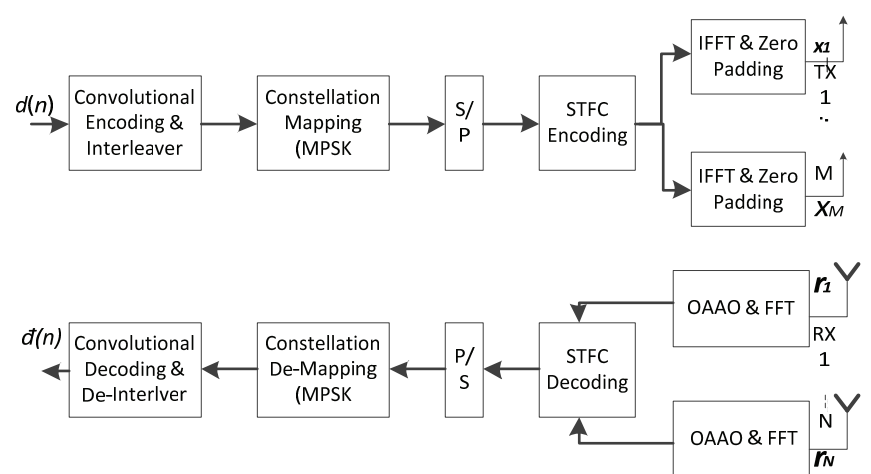

Fig. 2 STFC MB-OFDM UWB system.

elements are the $N_{f f t}$-point IFFT of the corresponding symbol vectors $\overline{\mathbf{x}}_{t, m}$ in $\mathbf{X}$. Hence, the transmitted matrix of STFC MBOFDM symbols is

$$
\begin{aligned}
\mathbf{X}_{O F D M}=\left\{\overline{\mathrm{x}}_{O F D M, t, m}\right\}_{T \times M} \\
=\left\{\operatorname{IFFT}\left\{\overline{\mathrm{x}}_{t, m}\right\}\right\}_{T \times M}
\end{aligned}
$$

The actual transmitted matrix is the matrix $\mathbf{X}_{Z P}$ whose elements are the $N_{f f t}$-length vectors $\overline{\mathrm{x}}_{O F D M, t, m}$ in $\mathbf{X}_{O F D M}$ appended with the 37-samples zero padded suffix (ZPS), denoted as $\overline{\mathrm{x}}_{Z P, t, m}$, before being transmitted to the channel. This means

$$
\mathbf{X}_{Z P}=\left\{\overline{\mathbf{x}}_{Z P, t, m}\right\}_{T \times M}
$$

The channels between $M$ TX antennas and $N \mathrm{RX}$ antennas are defined as the channel matrix $\mathbf{H}$

$$
\mathbf{H}=\left[\begin{array}{ccc}
\overline{\mathrm{h}}_{1,1} & \cdots & \overline{\mathrm{h}}_{M, 1} \\
\vdots & \ddots & \vdots \\
\overline{\mathrm{h}}_{1, N} & \cdots & \overline{\mathrm{h}}_{M, N}
\end{array}\right]
$$

where $\overline{\mathrm{h}}_{m, n}$ is the channel coefficient vector between the $m$-th TX antenna, for $m=1,2, \ldots, M$, and the $n$-th RX antenna, $n=$ $1,2, \ldots, N$, containing $l$ multipaths. The distribution and parameters of $\overline{\mathrm{h}}_{m, n}$ are determined by Eq. (1) and Table I for CM3, and by Eq. (2) and Table II for CM4.

In the receiver, the received signals at the $n$-th $\mathrm{RX}$ antenna during the $t$-th transmitted OFDM symbol duration is computed as

$$
\overline{\mathrm{r}}_{t, n}=\sum_{m=1}^{M}\left(\overline{\mathrm{x}}_{Z P, t, m} \otimes \overline{\mathrm{h}}_{m, n}\right)+\overline{\mathrm{n}}_{t, n}
$$

where $\otimes$ denotes the linear convolution, $\overline{\mathrm{x}}_{Z P, t, m}$ is the $t$-th transmitted MB-OFDM symbol including ZPS from the $m$-th TX antenna, and $\overline{\mathrm{n}}_{t, n}$ is the zero mean additive white Gaussian noise (AWGN) vector. The ZPS is removed by an OverlapAnd-Add-Operation (OAAO) prior to the FFT operation. The OAAO operates by taking $\left(\mathrm{N}_{f f t}+1\right)$ to $\left(\mathrm{N}_{f f t}+\mathrm{N}_{Z P S}\right)$ samples from the received symbols $\overline{\mathrm{r}}_{t, n}$, and adding them to the first $\mathrm{N}_{\mathrm{ZPS}}$ 
samples of the received symbols $\overline{\mathrm{r}}_{t, n}$. Then, the first $\mathrm{N}_{f f t}$ symbol will be used to decode the transmitted symbol, creating a circular convolution between the transmitted OFDM symbols before ZPS addition and the channel $\overline{\mathrm{h}}_{m n}[8]$. Hence, after performing OAAO, we have

$$
\overline{\mathrm{r}}_{O F D M, t, n}=\sum_{m=1}^{M} \overline{\mathrm{x}}_{O F D M, t, m} * \overline{\mathrm{h}}_{m, n}+\overline{\mathrm{n}}_{t, n}
$$

where (*) denote circular convolution. Using the same approach as [8], after the FFT block, the input signals of the STFC decoder is calculated as

$$
\overline{\mathfrak{r}}_{t, n}=\sum_{m=1}^{M} \overline{\mathrm{x}}_{t, m} \bullet \overline{\mathfrak{h}}_{m, n}+\overline{\mathrm{n}}_{t, n}
$$

where $(\bullet$ ) denotes the dot (Hardamard) product between the two vectors, $\overline{\mathfrak{r}}_{t, n}=\operatorname{FFT}\left(\overline{\mathrm{r}}_{O F D M, t, n}\right)=\left[\mathfrak{r}_{m, n, 1}, \ldots, \mathfrak{r}_{m, n, N_{f f t}}\right]^{T}$, $\overline{\mathrm{x}}_{t, m}$ is the original modulation symbols, $\overline{\mathrm{h}}_{m, n}=\mathrm{FFT}\left(\overline{\mathrm{h}}_{m n}\right)=$ $\left[\hbar_{m, n, 1}, \ldots, \hbar_{m, n, N_{f f t}}\right]^{T}, \quad$ and $\overline{\mathfrak{n}}_{t, n}=\operatorname{FFT}\left(\overline{\mathrm{n}}_{t, n}\right)$. Denote $\mathcal{R}=\left\{\overline{\mathfrak{x}}_{t, n}\right\}_{T \times N}$ to be received signals matrix after FFT, $\mathcal{H}=\left\{\overline{\mathfrak{h}}_{m, n}\right\}_{M \times N}$ to be the channel response matrix, and $\mathcal{N}=\left\{\bar{n}_{t, n}\right\}_{T \times N}$ the matrix of noise. Then we can re-write (9) as

$$
\mathcal{R}=\mathbf{X} \circ \mathcal{H}+\mathcal{N}
$$

where $\left({ }^{\circ}\right)$ denotes the operation which is similar to the normal matrix multiplication, except that each element in $\mathcal{R}$ is determined by (9), i.e. involving in the dot product between vectors.

We use the same approach as [8] for decoding the signals and detecting the transmitted data using Maximum Likelihood (ML) detection. The detected vectors are decided by

$$
\left\{\tilde{\overline{\mathbf{x}}}_{t, m}\right\}=\arg \min _{\left\{\overline{\mathrm{x}}_{t, m}\right\}}\|\mathcal{R}-\mathbf{X} \circ \mathcal{H}\|_{F}^{2}
$$

Since the matrix $\mathbf{X}$ preserves its orthogonality in the similar manner as in a conventional STBC MIMO system, the STFC MB-OFDM UWB system can also employ a simple linear decoding process. For simplicity, we can omit the time index $t$. Hence in the $2 \mathrm{I} 1 \mathrm{O}$ configuration with M-PSK modulation, we have the following decoding metrics

$$
\begin{aligned}
& \overline{\mathrm{x}}_{1}=\arg \min _{\overline{\mathrm{x}} \in \mathcal{C}^{N_{D}}}\left\|\left[\left|\left(\overline{\mathfrak{h}}_{1}^{*} \bullet \overline{\mathfrak{r}}_{1}+\overline{\mathfrak{h}}_{2} \bullet \overline{\mathfrak{r}}_{2}^{*}\right)-\overline{\mathrm{x}}\right| .^{\wedge} 2\right]\right\|_{F}^{2} \\
& \overline{\mathrm{x}}_{2}=\arg \min _{\overline{\mathrm{x}} \in \mathcal{C}^{N_{D}}}\left\|\left[\left|\left(\overline{\mathfrak{h}}_{2}^{*} \bullet \overline{\mathfrak{r}}_{1}-\overline{\mathfrak{h}}_{1} \bullet \overline{\mathfrak{r}}_{2}^{*}\right)-\overline{\mathrm{x}}\right|^{\wedge} 2\right]\right\|_{F}^{2}
\end{aligned}
$$

For the 2I2O, the decoding metrics are

$$
\begin{aligned}
\overline{\mathrm{x}}_{1}=\arg \min _{\overline{\mathrm{x}} \in \mathcal{C}^{N_{D}}} \|\left[\mid\left(\overline{\mathfrak{h}}_{11}^{*} \bullet \overline{\mathfrak{r}}_{11}+\overline{\mathfrak{h}}_{21} \bullet \overline{\mathfrak{r}}_{21}^{*}+\overline{\mathfrak{h}}_{12}^{*} \bullet \overline{\mathfrak{r}}_{12}+\overline{\mathfrak{h}}_{22}\right.\right. \\
\left.\left.\bullet \overline{\mathfrak{r}}_{22}^{*}\right)-\left.\overline{\mathrm{x}}\right|^{\wedge} 2\right] \|_{F}^{2} \\
\overline{\mathrm{x}}_{2}=\arg \min _{\overline{\mathrm{x}} \in \mathcal{C}^{N_{D}}} \|\left[\mid\left(\overline{\mathfrak{h}}_{21}^{*} \bullet \overline{\mathfrak{r}}_{11}-\overline{\mathfrak{h}}_{11} \bullet \overline{\mathfrak{r}}_{21}^{*}+\overline{\mathfrak{h}}_{22}^{*} \bullet \overline{\mathfrak{r}}_{12}-\overline{\mathfrak{h}}_{12}\right.\right. \\
\left.\bullet \overline{\mathfrak{r}}_{22}^{*}\right) \\
\left.-\left.\overline{\mathrm{x}}\right|^{\wedge} 2\right] \|_{F}^{2}
\end{aligned}
$$

where $N_{D}$ is number of data subcarriers $\left(N_{D}=100\right.$, according to the standard [22]), and $\mathcal{C}^{N_{D}}$ denotes the $N_{D}$-dimensional complex space of the transmitted vector $\bar{x}$. More importantly, each data point in an MB-OFDM symbol can be decoded separately rather than jointly [8], thus the decoding process is significantly simplified. In particular, the decoding metric of each data at the $k$-th subcarrier, for $\mathrm{k}=1, \ldots, N_{D}$, in the MBOFDM symbols are

$$
\begin{aligned}
& \tilde{\mathrm{x}}_{1, k}=\arg \min _{x_{1, k} \in \mathcal{C}}\left[\left|\left(\hbar_{1, k}^{*} \mathfrak{r}_{1, k}+\hbar_{2, k} \mathfrak{r}_{2, k}^{*}\right)-x_{1, k}\right|^{2}\right] \\
& \tilde{\mathrm{x}}_{2, k}=\arg \min _{x_{2, k} \in \mathcal{C}}\left[\left|\left(\hbar_{2, k}^{*} \mathfrak{r}_{1, k}-\hbar_{1, k} \mathfrak{r}_{2, k}^{*}\right)-x_{1, k}\right|^{2}\right]
\end{aligned}
$$

for the $2 \mathrm{I} 1 \mathrm{O}$ configuration, and

$$
\begin{aligned}
& \tilde{\mathrm{x}}_{1, k}=\arg \min _{x_{1, k} \in \mathcal{C}}\left[\left|\sum_{n=1}^{2}\left(\hbar_{1, n, k}^{*} \mathfrak{r}_{1, n, k}+\hbar_{2, n, k} \mathfrak{r}_{2, n, k}^{*}\right)-x_{1, k}\right|^{2}\right] \\
& \tilde{\mathrm{x}}_{2, k}=\arg \min _{x_{2, k} \in \mathcal{C}}\left[\left|\sum_{n=1}^{2}\left(\hbar_{2, n, k}^{*} \mathfrak{r}_{1, n, k}-\hbar_{1, n, k} \mathfrak{r}_{2, n, k}^{*}\right)-x_{2, k}\right|^{2}\right]
\end{aligned}
$$

for the 2I2O configuration. Generalization for the case of $M$ TX and $N$ RX antennas is straightforward, thus it is not mentioned in this paper due to the limited space.

\section{Simulation Results.}

The performance in terms of BER of the proposed system is assessed by Monte-Carlo simulations for three different configurations, i.e. SISO (single-input single-output), 2I1O and $2 \mathrm{I} 2 \mathrm{O}$, assuming perfect channel state estimations be available at the receiver. The total transmitted power from all TX antennas is kept equal at all times in all configurations, in order to fairly compare their performances. The channel conditions and their associate parameters are described in Section II. Channel coefficients are assumed to be constant during two consecutive OFDM symbols, i.e. during each Alamouti STFC block, but random between consecutive Alamouti STFC blocks. The channel realizations are simulated by the Matlab ${ }^{T M}$ program enclosed in the appendix of IEEE 802.15.6 channel modeling subcommittee final document [15]. The other simulation parameters are listed in Table III.

We compare the system performance for BPSK, QPSK, and 8PSK modulations in each channel model CM3 and CM4. In particular, we use QPSK modulation to simulate the effect of body directions in CM4 for both MIMO (2I1O and 2I2O) configurations. The number of OFDM symbols sent in each run of simulation is 50,000 in order to get at least 100 bit errors in every $E_{b} / N_{o}$ point. 
TABLE III. SIMULATION PARAMETERS.

\begin{tabular}{c|c}
\hline Parameters & Value \\
\hline FFT \& IFFT size $N_{f f t}$ & 128 \\
\hline Number of ZPS $N_{Z P S}$ & 37 \\
\hline Convolutional coder $(\mathrm{K}=7)$ rate & $1 / 2$ \\
\hline Conv. decoder and mode & Viterbi, Hard \\
\hline Interleaver/De-interleaver & $\begin{array}{l}\text { Column-wise written, } \\
\text { row-wise read }\end{array}$ \\
\hline Average number of paths in CM3 & 38 \\
\hline Average number of paths in CM4 & 400 \\
\hline Body directions & $0^{\circ}, 90^{\circ}, 180^{\circ}, 270^{\circ}$ \\
\hline
\end{tabular}

The performances of the proposed STFC MB-OFDM and of the conventional (SISO) MB-OFDM system for CM3 channels, with QPSK modulation, are compared in Fig.3. The average transmitted powers are kept to be the same for both systems, by scaling down the STFC MB-OFDM's power by a factor of 2. The simulation results show that the improvement of at least $5 \mathrm{~dB}$ and $10 \mathrm{~dB}$ can be achieved at BER $=10^{-4}$ in the $2 \mathrm{I} 1 \mathrm{O}$ and 2I2O STFC MB-OFDM systems, respectively, compared to the conventional MB-OFDM. Thus, the implementation of the proposed STFC concept in MB-OFDM provides superior performance over the conventional MBOFDM, thanks to the higher diversity order introduced by the STFC (see [8] for more detail).

Fig.4 compares the performance of the STFC MB-OFDM UWB system in CM3 for BPSK, QPSK, and 8PSK modulations, for both MIMO configurations. It clearly shows that the system can achieve a good BER performance from a reasonably low $E_{b} / N_{o}$ range in both MIMO configurations, even if we use 8PSK modulation. For example, a BER of $10^{-4}$ can be attained at $E_{b} / N_{o}=4.8 \mathrm{~dB}$ for 2I2O-QPSK, $8.5 \mathrm{~dB}$ for 2I2O-8PSK, $10.2 \mathrm{~dB}$ for $2 \mathrm{I} 1 \mathrm{O}-\mathrm{QPSK}$, and $14 \mathrm{~dB}$ for $2 \mathrm{I} 1 \mathrm{O}-$ 8PSK. It is due to the fact that $N_{Z P S}=37$ is close enough to the mean number of multipath, i.e. $\bar{L}=38$ (cf. Table I), in which the system experience frequency flat fading channels. Significant improvement of at least $6 \mathrm{~dB}$ is shown by employing $2 \mathrm{I} 2 \mathrm{O}$, compared to $2 \mathrm{I} 1 \mathrm{O}$ at $\mathrm{BER}=10^{-4}$. Whereas, the system performance improves by $2-4 \mathrm{~dB}$, if we employ lower level M-PSK modulations.

Fig.5 depicts the system performance in CM4 in the case of $0^{\circ}$ body direction for different MIMO configurations and modulation schemes. In this very dispersive channel, severe performance degradations occur if we move to higher level MPSK modulations. It can be seen that the 2I2O configuration provides a huge advantage in terms of BER for a given $E_{b} / N_{o}$, compared to the $2 \mathrm{I} 1 \mathrm{O}$ configuration. The figure also shows a flattened performance (error floor) after $\mathrm{Eb} / \mathrm{No}=10 \mathrm{~dB}$ for all configuration and modulation schemes. This will be explained in detail later.

The effect of body directions to the system performance in CM4 is depicted in Fig.6 for QPSK modulation. The front body $\left(0^{\circ}\right.$ direction $)$ has a LOS component, which results in better performance compared to other directions of the body. The back of the body $\left(180^{\circ}\right.$ direction) only receives NLOS signals. Nonetheless, the system is still able to cope with the very dispersive channel and produce a significant performance, particularly with the $2 \mathrm{I} 2 \mathrm{O}$ configuration. The performance degrades $6 \mathrm{~dB}$ for $\mathrm{BER}=10^{-4}$, compared to the front body. Interesting results arise from the right-side $\left(90^{\circ}\right.$ direction) and left-side $\left(270^{\circ}\right.$ direction $)$ of the body. The degradation of the performance is due to the fact that they are only able to receive partially LOS signals through the antenna's side lobes, as well as multipath signals. This observation can also be seen clearly in Table II.

From Figures 5 and 6, error floors, where increasing $E_{b} / N_{o}$ further does not bring about a significant improvement, can be observed. This is due to the fact that the ZPS is much shorter than the channel length in the very dispersive channel CM4, hence the ISI cannot be overcome completely. The residual ISI is still large enough to neutralize the performance. This is the reason why the MB-OFDM technique has been proposed where consecutive MB-OFDM symbols are transmitted over different RF frequencies, thus avoiding the residual ISI. For simplicity, simulations are run here in the baseband, rather than in the RF band, thus the error floor can be observed. In other words, the performance provided in the paper works as the lower bound for the improvement that could be provided by the proposed system.

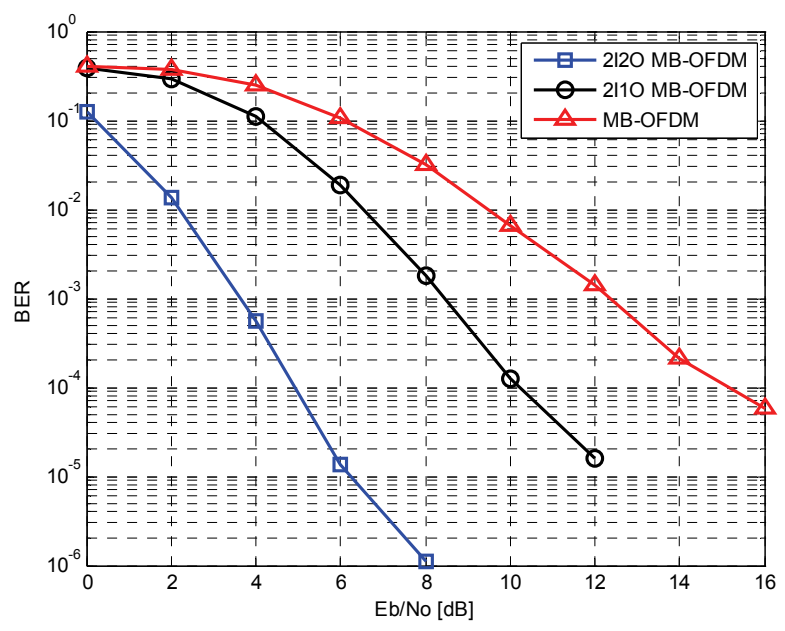

Fig.3. Performance comparison of the STFC MB-OFDM and conventional MB-OFDM systems in CM3 WBAN channels.

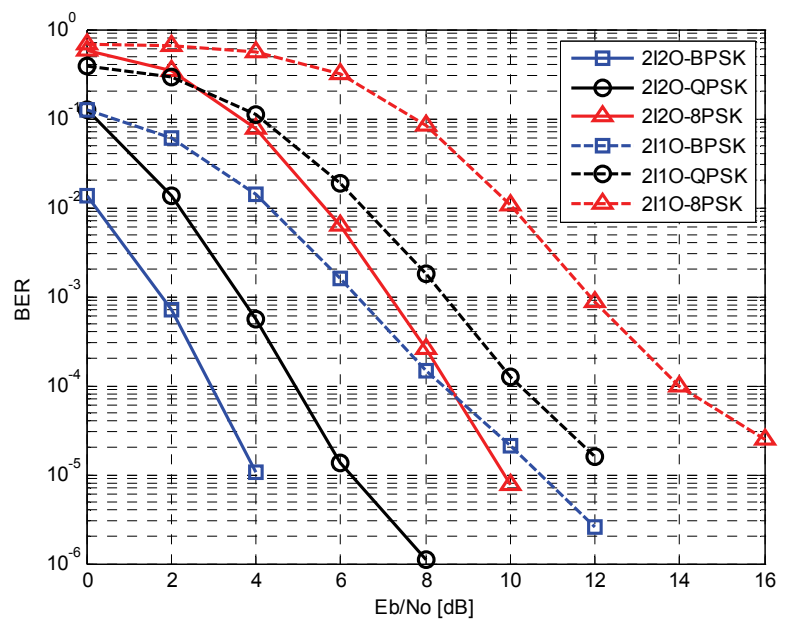

Fig.4. Performance of STFC MB-OFDM in CM3 with M-PSK modulations. 


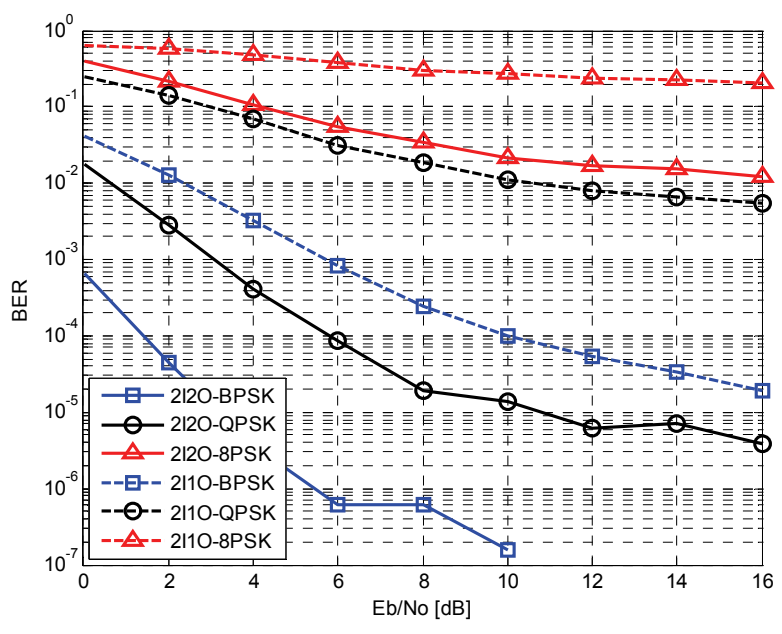

Fig.5. Performance of STFC MB-OFDM in CM4, with M-PSK modulations.

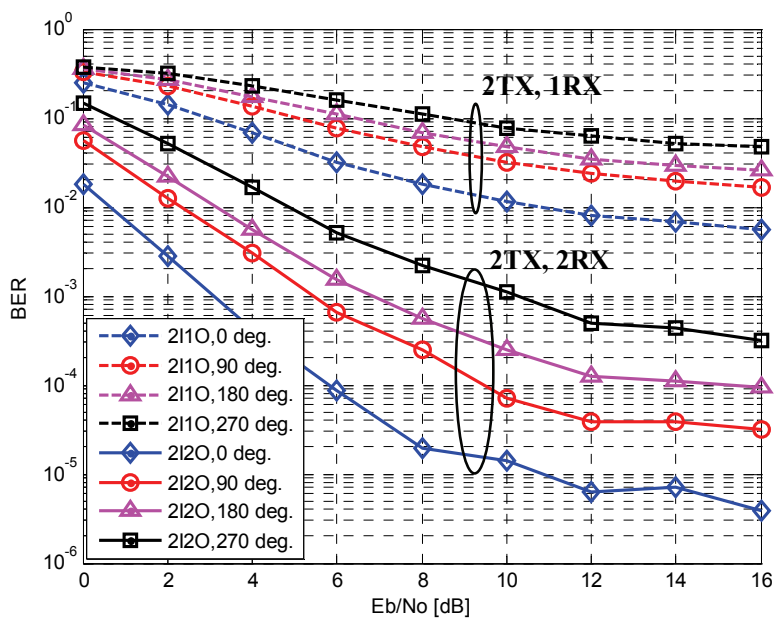

Fig.6. Performance of STFC MB-OFDM in CM4 for various body directions, with Q-PSK modulation.

\section{CONCLUSION}

We have presented the performance analyses of the STFC MB-OFDM UWB system in WBAN channels. Simulation results confirm that the proposed system can achieve significantly better BER performances in the order of 5-10 dB, in both MIMO configurations, compared to the conventional MB-OFDM system. With the same MIMO configuration, the system performance improves by $2-4 \mathrm{~dB}$ if we employ lower level M-PSK modulations. Moreover, though the body direction affects the performance significantly due to the availability of LOS or partial LOS paths, the proposed system is still more robust, compared to the conventional MB-OFDM one. We conclude that the proposed system can provide a robust, high data rate transmission for WBAN applications. Our future works would be implementing higher-order STFCs in WBAN channels.

\section{ACKNOWLEDGMENT}

The first author is grateful to the AusAID who provides financial support through the Australian Development
Scholarship (ADS) scheme. The authors also would like to thank the anonymous reviewers for insightful feedbacks.

\section{REFERENCES}

[1] S. M. Alamouti, "A simple transmit diversity technique for wireless communications," IEEE Journal on Selected Areas in Communications, vol. 16, no. 8, pp. 1451-1458, Oct. 1998.

[2] A. F. Molisch, M. Z. Win, and J. H. Winters, "Space-time-frequency (STF) coding for MIMO-OFDM systems," IEEE Communication Letter, vol. 6, no. 9, pp. 370-372, Sept. 2002.

[3] M. Fozunbal, S. W. McLaughlin, and R. W. Schafer, "On space-time frequency coding over MIMO-OFDM systems," IEEE Trans. Wireless Communication, vol. 4, no. 1, pp. 320-331, Jan. 2005.

[4] W. P. Siriwongpairat, et al., "Multiband-OFDM MIMO Coding Framework for UWB Communication Systems", IEEE Trans. Signal Processing, vol. 54, no. 1, Jan. 2006.

[5] T.H. Tan and K.C. Lin," Performance of Space-Time Block Coded MBOFDM UWB Systems", in Proc. IEEE 4th Annual Communication Networks and Services Research Conference CNSR'06, pp.323-327, May 2006.

[6] Q. Yang, and K. S. Kwak, "Outage performance of STBC MB-OFDM UWB”, Int. Journal on Electronic Communication, vol.63, issue 8, pp: 685-688, Aug. 2008.

[7] L.C. Tran et al., " Space Time Frequency Code in MB-OFDM UWB Communications: Advanced order-8 STFC and its performance", International Symposium on Communication and Information Technology, pp. 380-385, Oct. 2007.

[8] L.C. Tran and A. Mertins, "Space-Time-Frequency Code Implementation in MB-OFDM UWB Communications: Design Criteria and Performance", IEEE Trans. Wireless Communication, vol. 8, no. 2, Feb. 2009.

[9] L.C. Tran, et al., "Unitary Differential Space-Time-Frequency Codes for MB-OFDM UWB," in Proc. of $9^{\text {th }}$ IEEE International Symposium on Communication and Information Technology, pp. 1161-1166, Sep. 2009.

[10] L. C. Tran, A. Mertins, and T. A. Wysocki, "Quasi-orthogonal spacetime-frequency codes in MB-OFDM UWB", Journal on Computers and Electrical Engineering, vol. 36, issue 4, pp. 766-774, July 2010.

[11] J. Foerster et al., "Channel modeling sub-committee report final," IEEE P802.15-02/490r1-SG3a, Oct. 2005.

[12] J. Takada, et al., "Static Propagation and Channel Models in Body Area", COST 2100 TD(08)639, Oct. 2008.

[13] K.Y. Yazdandoost, and K. Sayrafian-Pour, "Channel Model for Body Area Network (BAN)", IEEE 802.15-08-0033-05, Sept. 2008

[14] Y. Wang, et al., "Characterization of the Indoor Multi-antenna Body-toBody Radio Channel”, IEEE Trans. Antennas and Propagation, vol. 57, no. 4, April 2009.

[15] K.Y. Yazdandoost , et al. , “ Channel Model for Body Area Network (BAN) “, IEEE P802.15-08-0780-12-0006, Nov. 2010.

[16] M. Chen, et al., "Body Area Networks: A Survey", Springer Journal on Mobile Netw Appl, vol. 16, no. 2, pp.171-193, Aug. 2010.

[17] Q. H. Abbasi, et al., "Characterization and Modelling Of Ultra Wideband Radio Links For Optimum Performance Of Body Area Network In Health Care Applications" in Proc. of the 2011 IEEE International Workshop on Antenna Technology, pp. 206-209, Mar. 2011.

[18] Q. H. Abbasi, et al., "Radio Channel Characterization and OFDM-based Ultra Wideband System Modelling for Body-Centric Wireless Networks", in Proc. of 2011 International Conference on Body Sensor Networks, pp. 89-94, July 2011.

[19] Q. H. Abbasi, et al., "Experimental Characterization and Statistical Analysis of the Pseudo-Dynamic Ultra wideband On-Body Radio Channel", IEEE Antennas and Wireless Propagation Letters, pp. 748751, vol. 10, July 2011.

[20] M. M. Khan, et al., "Investigation of Body Shape Variations Effect on the Ultra-Wideband On-Body Radio Propagation Channel", in Proc. of 2011 International Conference on Electromagnetic in Advance Applications (ICEAA), Italy, Sept. 2011.

[21] H. Schulze and C. Luders, Theory and Application of OFDM and $C D M A$, John Wiley \& Son, Chichester, 2005.

[22] A. Batra et al., "Multiband OFDM physical layer specification," WiMedia Alliance, Final Deliverable 1.5, Aug. 2009. 